\title{
Historia y sus métodos; el problema de la metodología en la investigación histórica
}

\author{
History and its methods; the problem of methodology \\ in historical research
}

\author{
Izabela Tkocz* \\ Jesús Adolfo Trujillo Holguín**
}

\begin{abstract}
*Docente en las Facultades de Artes y Filosofía y Letras de la Universidad Autónoma de Chihuahua (México). Es Maestra en Humanidades con especialidad en historia e historiografia. Es de nacionalidad polaca y también colabora en la Universidad La Salle. Tiene participaciones como ponente en coloquios de investigación de la UACH y congresos nacionales e internacionales. Publicó el capítulo "La propaganda socialista soviética y su influencia en la cultura de Chihuahua en las décadas de 1920 a 1940 " en Acercamientos a la historia de la educación. Diálogos, actores y fuentes en la construcción del conocimiento histórico (2017) y el capítulo "Reforma socialista al artículo tercero constitucional de 1934; análisis de su contexto histórico” en el libro El artículo tercero en cien años de la Constitución Política de 1917 (2018). Actualmente cursa el Doctorado en Educación, Artes y Humanidades. Correo electrónico izatkocz@hotmail.com

(D) htp://orcid.org/0000-0002-3973-2888

**Profesor investigador de la Universidad Autónoma de Chihuahua (México). Es doctor en educación y cuenta con una especialidad en competencias docentes por la Universidad Autónoma de Madrid (España). Entre sus publicaciones recientes están: Debate legislativo y educación. El artículo tercero a cien años de la Constitución Política de 1917 (coord.) (2018). Cuenta con reconocimiento al perfil Prodep y del Sistema Nacional de Investigadores Nivel 1. Es socio del Consejo Mexicano de Investigación Educativa, de la Sociedad Mexicana de Historia de la Educación y de la Red de Investigadores Educativos Chihuahua. Sus temas de interés son la historia e historiografía de la educación y formación de maestros. Correo electrónico jatrujillo@uach.mx
\end{abstract}

https://orcid.org/0000-0002-6738-1878

Historial editorial

Recibido: 25-mayo-2018

Primera revisión: 30-mayo-2018

Segunda revisión: 02-septiembre-2018

Aceptado: 19-septiembre-2018

Publicado: 31-octubre-2018 
Historia y sus métodos; el problema de la metodología en la investigación histórica

Resumen

El presente artículo tiene como objetivo enfocarse en la problemática de la metodología de la investigación histórica, la cual comprende los estudios del pasado como parte de las humanidades, donde se interrelacionan varias disciplinas, en algunos casos difíciles de separar o con límites imprecisos. La temática de la metodología de investigación histórica está relacionada con el trabajo dentro de la tesis doctoral, realizada en el Posgrado de la Facultad de Filosofía y Letras en la Universidad Autónoma de Chihuahua, titulada "La influencia de la propaganda socialista soviética en la cultura y educación durante la década de I920 a 1930 en Chihuahua" bajo la dirección del doctor Jesús Adolfo Trujillo Holguín. Desde el inicio de la investigación surgió el problema para trabajar con las fuentes primarias, en relación a la veracidad de los datos encontrados, la verificación de la autenticidad, su contextualización y -finalmente- en el proceso de reflexión e interpretación. En la búsqueda de la metodología o metodologías que ayudaran a superar las dificultades, se revisaron propuestas de diferentes autores -entre quienes destacan Jenkins, Topolski, Gotschalk y Handelsman ${ }^{\mathrm{I}}-\mathrm{y}$ algunas maneras que proponen para solucionar el problema. Finalmente se optó por dividir los métodos en dos partes: una que permitiera seleccionar y verificar los datos y otra que se adecuara a la interpretación de las fuentes primarias. En el presente trabajo se exponen las metodologías usadas y su justificación.

Palabras clave: Historia e historiografía, investigación, metodología de la investigación, historia crítica.

History and its methods; the problem of methodology in historical research. 


\begin{abstract}
The aim of this article is to describe the problems inherent to the methodology of historical research. Historical research studies the past, interrelating several disciplines, however, the boundaries between disciplines are difficult to separate and locate. This is a doctoral thesis of historical research, entitled: "The influences of Soviet Socialist Propaganda on Culture and Education in the 20 s and 30 in Chihuahua". The study is conducted at the Postgraduate program of Facultad de Filosofía y Letras at the Universidad Autónoma de Chihuahua and the doctoral thesis advisor is Dr. Jesús Adolfo Trujillo Holguín. We describe here several problems that have arisen from the beginning of the study. These problems range from working with primary sources, to verifying the veracity of the data bound and contextualizing the data. The most challenging part of the process, however, has been data reflection and interpretation. In the search of the methodology or methodologies that would help to solve the research problem, we reviewed the work of different authors including Jenkins, Topolski, Gotschalk and Handelsman. Finally, we chose to use two different methodologies: the first methodology would allow us to select and verify the data and the second would allow the interpretation of the primary sources. The intention of the present article is to describe and justify the methodologies used in the study.
\end{abstract}

Keywords: History and historiography, investigation, research methodology, critical history. 


\section{Introducción}

Uno de los elementos más importantes que componen la cultura metodológica del historiador es el conocimiento acerca de los límites y uso de la metodología. De este conocimiento depende la calidad del trabajo de investigación, que se supone debería ser proporcional a la cultura metodológica que se manifiesta en la conciencia individual y en el uso práctico de los métodos de investigación. En otras palabras, a más alta cultura metodológica del historiador más alta calidad del conocimiento que proporciona acerca del pasado.

La historia es parte de las humanidades y por lo tanto no se puede limitar solo al conocimiento histórico y a los métodos usados por los historiadores. Es necesario interactuar con otras disciplinas tales como la filosofía, geografía, sociología, economía, arqueología, antropología, psicología, entre muchas otras. Handelsman (2017) menciona que:

(...) las ciencias auxiliares de la historia tienen que proporcionar al historiador las herramientas que le permitan hábilmente buscar, investigar y reflexionar. En otras palabras, actuar críticamente analizando la fuente histórica, para poder descalificar o confirmar la utilidad de la información y al mismo tiempo usar esta información para conocer el pasado y poder analizarlo (p. 73). ${ }^{2}$

La tarea del historiador es estudiar el pasado del ser humano como individuo y como parte de la sociedad. Por lo tanto, es todavía más importante el uso de la metodología o metodologías adecuadas al tema y tipo de la investigación. Hay también diferentes tipos de historia: cultural, social, económica, militar, del arte, de la educación, de la propaganda, política, medieval, entre otras. Dentro del contexto que se 
presenta parece ser indispensable buscar la o las metodologías que permiten validar el trabajo del historiador.

A lo largo de la historia, los modelos de investigación histórica cambiaron. Siempre ocurrió que uno se hacía más importante que otro, sin que alguno se desvaneciera por completo. Cada modelo aportaba los valores definidos a la investigación. Algunas veces -cuando todavía predominaba el modelo concreto- se aparecían los precursores del siguiente. En la antigua Grecia, donde apareció Herodoto, Finley ${ }^{3}$ demostró que la conciencia histórica era un mito que se expresaba en la poesía, no en la literatura histórica (Finley, 1982). No es coincidencia que la mayoría de las grandes obras históricas de la Antigüedad fueran en realidad ensayos o introducciones a la política, llenas de mensajes.

Cuando pasó el periodo de los grandes historiadores de la Edad Antigua como Tucidides o Tacito, con quienes no se podría competir, apareció la historiografía enferma de la Edad Media. La tradición de las crónicas y annales necesitó siglos para aportar a la narración histórica la conciencia de las nuevas tareas (Topolski, 1998).

Laboriosamente se formó el modelo de la nueva narración histórica. La búsqueda de la verdad -acentuada dentro del siglo XVIII- se convirtió en la tarea de todos los historiadores. Se empezó a establecer el modelo crítico de la historia. En el siglo XIX y XX se dio la expansión de los diferentes conceptos de la historia: Hegel, Marks, Ranke, Comte, Dilthey, Croce, Collingwood, Bloch, Braudel, Benjamin, Le Goff, White y muchos otros.

El investigador debería también tomar en cuenta las discusiones del concepto de posmodernidad y las críticas que se hacen a la historia como la verdad de nuestro pasado. Los principales autores posmodernos como Nietzche, Wittgenstein o Derrida consideran que la 
verdad es producto de nuestro discurso, por lo tanto, es relativa y depende de la posición de quien la anuncia. Rorty menciona -y esta visión también la comparte Jenkins- que la verdad está íntimamente relacionada con el poder. En el sentido más amplio, el poder se puede entender a través de la figura del historiador, porque es él que genera conocimiento y por medio de este supuestamente reflexión y conciencia. El conocimiento siempre depende del contexto social, es hecho por alguien para alguien (Bolaños de Miguel, 20II). La historia, según Jenkins (2006), no es en absoluto un fenómeno natural ni tiene nada eterno, algo con lo que muchos historiadores están de acuerdo. En otras palabras, la historia parece ser un discurso continuo, no absoluto. Jenkins menciona que "El pasado como historia siempre ha estado y siempre estará necesariamente configurado, envuelto en tropos, figurado en tramas, leído, mitificado e ideologizado en formas que nos resulten convenientes" (p. 132). Steiner le llama las imágenes del pasado (Steiner, 2006).

Hablando entonces de la verdad histórica como absoluta es imposible, pues como seres humanos nos formamos en diferentes ambientes, sea culturales o ideológicos, por lo tanto, las visiones e interpretaciones de la historia son distintas. Al ser subjetivas es fácil descalificarlas y por lo tanto es necesario enfocarse en la metodología, para que sean por lo menos verificables. Parece entonces que la llave de la investigación histórica es la metodología.

La división de las ciencias es muy grande y en diferentes tiempos y

122 épocas se cambió el trato. Ya Aristóteles las dividía en tres: las teóricas (conocimiento), prácticas (reglas de actuar) y fabricadas (reglas de crear). Hoy en día, las ciencias generalmente se dividen en formales, empíricas y sociales. Todavía se discute cómo clasificar la ciencia y hay dudas al respecto. Por ejemplo, la historia ha sido calificada como parte 
de las humanidades, pero la enseñanza de la historia como ciencia empírica (Rybinska, 20I5).

La historia de la ciencia dice que la característica del conocimiento científico, que se basa en la experiencia, es principalmente su inestabilidad. Kuhn introdujo el concepto de revolución científica como la etapa de desarrollo de la ciencia que conduce al cambio de paradigma (Kuhn, I962). Por lo tanto, en lugar de la verdad se estableció la idea de la alta probabilidad, que dentro de la historia debería interpretarse como uno de los muchos acercamientos al problema para comprender e interpretar el fenómeno. Dentro de la problemática presentada parece importante abracar el concepto de explicatum (dato que se debe aclarar) y explicans (explicación del dato) que manejaba Karl Popper (Popper, 1969). Para que la explicación fuera válida, tiene que suceder la relación lógica entre sus componentes. También Popper subrayaba que la hipótesis tiene que ser falsaciable. Entre otros postulados que le parecieron importantes se encuentra la simplicidad y habilidad de predecir otros fenómenos (Borleigh, I983).

El rol del historiador es la interpretación de las fuentes. El conocimiento se basa en datos concretos, pero existe la división en la ciencia popular y académica. Entonces para poder formar su opinión, reflexionar e interpretar, el investigador necesita conocimientos muy amplios.

\section{Metodología de la historia}

La base del presente artículo es el trabajo de investigación titulado "La influencia de la propaganda socialista soviética en la cultura y educación durante la década de $\mathbf{1 9 2 0}$ a 1930 en Chihuahua”. El periodo que abarca implicó que desde el inicio se contemplara trabajar con 
fuentes primarias e incorporar como soporte teórico a las fuentes secundarias. Era necesario esclarecer el concepto de cada una y la manera en que serían tratadas.

Uno de los principales historiadores del siglo XX (Hobsbawm, I998) menciona que en primer lugar "todo trabajo histórico entraña hacer una selección, una minúscula selección, de algunas cosas, partiendo de la infinidad de actividades humanas del pasado y de lo que afectó a tales actividades" (p. 73). Podría decirse -entonces- que la investigación histórica comienza por la puntualización del tema a investigar. Como en cualquier investigación y como en cualquier disciplina científica, el primer momento metodológico es la delimitación o construcción de un objeto de estudio. Este proceso surge de la simple observación empírica (Klappenbach, 20I4). La primera fase metodológica de la investigación histórica -inspirada en Hobsbawm- no se aleja en lo esencial de lo planteado por Karl Popper en su tesis sobre la lógica en las ciencias sociales (Popper, I962).

También fue vital comprender qué es el trabajo de investigación con enfoque histórico y qué tareas tiene que realizar el historiador para manejar las fuentes primarias y otras evidencias a partir de las cuales se interpretan y reinterpretan los fenómenos pasados. Para realizarlo, es necesario dividir en las siguientes etapas:

- Localización y recopilación de fuentes primarias - en su mayoría documentales (la fase heurística).

- Crítica interna y externa de las fuentes encontradas.

- Producto final de historiografía: la síntesis (Gotschalk, I950).

El conocimiento histórico necesita de la utilización de las fuentes, sean documentos, testimonios u objetos y cumplen tres aspectos: primero, 
como base desde la que se construye el conocimiento histórico (fundamentación), segundo como medio por el que se transita para construir dicho conocimiento (demonstración) y tercero, como límite, en el sentido que pone una frontera al historiador, impidiéndole que pueda elucubrar sin argumentos acerca de su tema de investigación (verificación). La fundamentación se refiere a la fuente histórica de la que parte el investigador; la demonstración es el trayecto que se sigue en la investigación, ya que no basta con tener una base sólida, sino que se necesita desarrollar adecuadamente la investigación, la correcta interpretación de las fuentes consultados. La verificación se refiere a que una vez concluida la investigación histórica, deberá ponerse a consideración del lector el lugar exacto de donde se obtuvieron las fuentes consultadas, esto permitirá la confiabilidad necesaria y la suficiente certeza de que los resultados son producto de un trabajo serio y científico (Hurtado, s/f).

Teniendo claros los procedimientos generales, el trabajo se enfocó en la búsqueda de una o varias metodologías que auxiliaran el proceso de investigación. Por razones prácticas ${ }^{4}$ uno de los principales libros en que se basó el trabajo de investigación es Metodología de la historia de Jerzy Topolski. En dicho libro -que cuenta con más de 500 páginas- el autor reconstruyó el pensamiento de Marks y tomó los conceptos de la lógica formal, metodología de la ciencia, de la teoría de la comunicación y de la cibernética5 ${ }^{5}$ (Topolski, 1998).

En el texto se establecen tres conceptos básicos que describen el término Historia:

I. Es el conjunto de actividades que realizan los historiadores.

2. Es el resultado de esas actividades.

3. Es el pasado que se estudia, objeto de la investigación. 
Desde el inicio, divide la metodología de la historia en dos partes: pragmática y apragmática. La primera se distingue por:

I. Elección del campo de investigación.

2. Planteamiento de una cuestión en este campo.

3. Establecimiento de las fuentes en las que se basará el estudio (si la cuestión planeada va a tener respuesta).

4. Crítica (externa e interna) de las fuentes.

5. Descripción -siempre selectiva - de lo que ocurrió y de aquello a lo que se refiere la pregunta.

6. Explicación (¿por qué ocurrió así?).

7. Consecución de las premisas teóricas.

8. Formulación sintética de los resultados (es decir, respuesta a la pregunta dentro del terreno en consideración).

9. Valorización de las personas y los sucesos del pasado (Topolski, I998, p. 39). ${ }^{6}$

Por su parte, la metodología aprágmatica abarca generalidades históricas, afirmaciones históricas, leyes y afirmaciones estrictamente generales y el concepto de narración.

Para la aplicación de la metodología aprágmatica de la historia es indispensable, por lo tanto, adoptar, como se ve, los conceptos de: I) afirmación histórica; 2) generalización histórica; 3) ley, en el sentido semántico del terminó; 4) ley científica; 5) juicio de valor; 6) narración histórica; 7) lenguaje objeto y metalenguaje; 8) verdad; 9) isomorfismo ${ }^{7}$ (Topolski, I998, p. 4I). 
Para Topolski la historia como estudio del pasado debe utilizar como herramienta la metodología de la historia, a la cual dedicó la tercera parte de su libro. En él menciona que:

(...) el objeto de la metodología de la historia es la elaboración del campo general y específico como modelo de la ciencia en el sentido semántico, que tiene que servir para la separación de los datos verdaderos de los falsos. En seguida tiene que entregar las directivas heurísticas para la investigación y finalmente es necesario elaborar términos teóricos para la descripción del campo (I998, p. 29).

La definición específica de la investigación permite evitar la pérdida de tiempo. Por ejemplo, la narración que se considera como leyenda es también un dato histórico. Según Topolski, en la historia no se puede hablar solamente sobre la verdad o falsedad del dato, sino también sobre verdad o falsedad de la narración. Es importante recordar que en esta última la suma de datos verdaderos no significa que la imagen general es también verdadera. Se le puede describir con la ecuación siguiente:

$$
\mathrm{d} 1=\mathrm{V}, \mathrm{d} 2=\mathrm{V}, \mathrm{d} 3=\mathrm{V} ; \sum d \neq \mathrm{V}^{8}
$$

Donde $d$ significa el dato y $V$ su verdad. En la narración puede suceder también que aparte de los datos verdaderos existan algunos falsos y de todas formas no cambia la imagen general de la situación.

Los positivistas consideraban que la historia en el sentido de los fenómenos pasados es un mosaico de datos históricos que son algo primordial y primitivo para estudiar la materia, mientras que la narración de aquello es la fiel reflexión de la realidad. Topolski (I998) rechaza esta postura y para él: 
La realidad histórica es tan complicada y rica en su diversidad y conexiones que la construcción de los datos es indispensable para conocerla. En el enfoque dialéctico sucede el proceso constante de confrontación de la cada vez más conocida realidad histórica, a partir de los datos construidos. Esto significa que, basándose en la creciente y cambiante información, modificamos nuestra construcción. Las hipótesis débiles se hacen cada vez más fuertes y mejor argumentadas (p. I5I).

La realidad histórica como proceso -menciona Topolski- tiene las regularidades que investiga el historiador. Los resultados de la investigación son las reglas históricas. En la discusión acerca de si la historia es la ciencia ideográfica o nomotética es a favor de la última. Distingue tres tipos de reglas que manejan el proceso histórico: sincrónicas, diacrónicas y sincrónico-diacrónicas. Como realidad sincrónica considera la regla según la cual "ninguno de los elementos de la macro estructura -como es la realidad social- puede existir por separado, solo en la conexión sincrónica" (I998, p. 193). Las regularidades diacrónicas se comprueban en las causas de los fenómenos que provocan la existencia de otros. Las reglas sincrónicodiacrónicas se manifiestan dentro de las reglas del desarrollo. Habla también de libre voluntad, rechaza totalmente el fatalismo y subraya que el ser humano posee determinado margen de la libertad, por lo tanto, se puede considerar como creador de la historia, aunque está limitado por la geografía y la sociedad. La primera coarta la libre voluntad del ser humano ya que él es parte de la naturaleza. La limitación social consiste en que el hombre actúa y toma decisiones en las condiciones que ya existes y que no escogió. Igualmente pertenece a una determinada clase y grupo social.

Topolski diferencia el conocimiento histórico en dos tipos: el conocimiento directo e indirecto. El primero está basado en la 
observación física de los objetos existentes, como las armas, textiles, monumentos o documentos. El historiador conoce también cuando observa "los seres humanos reflejan más o menos las experiencias transmitidas de generación en generación" (Topolski, I998, p. 217). Cuando el investigador trata de leer el documento -o cualquier otro tipo de escritura- el conocimiento es indirecto, pues ya está usando como mediador al autor de dicho texto.

Todo el conocimiento sobre los hechos históricos que viene de las fuentes se le llama conocimiento del dato y está constituido por los hechos básicos. Dentro de las fuentes son datos aceptados, por ejemplo, porque son mencionados por un autor de confianza o porque se obtuvieron de otro lugar fuera de la fuente.

Para caracterizar el conocimiento fuera de la fuente del historiador, Topolski lo divide en popular (no académico) y académico. Al primero pertenecen los conocimientos del comportamiento humano, de la época, del mundo en general. El conocimiento académico comprende los datos de otras disciplinas y el conocimiento histórico y metodológico. El conocimiento fuera de la fuente se compone de observaciones y teoría. En esta división no es necesario separar ni ser estricto. Considera que todas las observaciones están equiparadas en sentido teórico y lógico.

El término de conocimiento fuera de la fuente se puede entender en el sentido actual o potencial. El primero es todo lo que sabemos fuera: I. De la información sobre los hechos históricos y 2. De la información que saquemos de ellos.

En el sentido potencial, el conocimiento fuera de la fuente es todo lo que podemos saber: I. De la información sobre los hechos históricos y 2. De la información que saquemos sobre los hechos históricos. 
Las frases todo lo que sabemos y todo lo que podemos saber están relacionadas con un investigador o con un grupo. Por lo tanto, el concepto de conocimiento fuera de la fuente es relativo. Se le puede relativizar también cuando hablamos de un problema.

En el caso de la explicación histórica se puede interpretar de diversas maneras:

I) Explicación por la descripción (descriptiva);

2) Explicación por indicación del origen de un fenómeno concreto (genética);

3) Explicación por la indicación del lugar de un fenómeno en una estructura dada (estructural);

4) Explicación por indicación de una causa (casual) (Topolski, I998, p. 4I3).

Topolski considera que el lenguaje (narración) es muy importante en la investigación histórica porque incluye vocabulario, gramática y funciones semánticas de las palabras; mismas que "atribuyen significados específicos a las palabras y a las oraciones. Para usar un lenguaje concreto correctamente, hay que conocer no solo el vocabulario y la gramática, sino también sus reglas semánticas" (I998, p. 473).

Para Łobocki (2005) la interpretación de los textos se puede hacer con dos métodos: a través de la fenomenología y de la heurística. El primero permite "entrar a la profundidad de la realidad." (p. 2I2)

La fenomenología puede servir como método para conocer el fenómeno, como postulaban Scheller e Ingarden, ${ }^{\text {IO }}$ porque permite regresar a las cosas como son en sí para conocerlas en profundidad. 
Gracias a la fenomenología, el texto deja de ser algo material y empieza a conducir al investigador para guiarlo hacia lo desconocido. Por lo tanto, lo más importante debería ser encontrar el sentido o la idea del texto (Domanska, 20Io).

Por su parte, la Heurística ${ }^{\text {II }}$ se entiende como la técnica de explicar, presentar y reflexionar sobre los textos. En un nivel más amplio, es el arte de comprender el sentido de diferentes planos de la realidad (Wysocka, 2004).

Krüger (2005) en su libro Introducción a la teoría y metodología de la pedagogía ${ }^{22}$ menciona:

a) El investigador debería presentar su posicionamiento al respecto del problema, después de conocer la literatura sobre el tema;

b) La primera presentación del problema debería ser constantemente verificada con las fuentes y eventualmente modificada;

c) El investigador debe preguntarse sobre el significado de las palabras y formas del texto, haciendo el análisis semántico;

d) La interpretación hermenéutica implica tomar en cuenta la situación que se presentó cuando el texto era elaborado;

e) La interpretación del texto tiene que auxiliarse de las fuentes adicionales, por ejemplo, con el análisis histórico-social;

f) En la investigación sobre la argumentación del texto también se toman en cuenta las cuestiones sintácticas; 
g) El investigador sistemático tiene que dividir el texto para comprobar la hipótesis e interpretar;

h) El interpretador debe revisar críticamente el texto, el pensamiento del autor, fijándose que la argumentación sea lógica y clara;

i) Las explicaciones e interpretaciones deben ubicarse dentro del contexto social y cultural de determinado periodo histórico, a fin de poder comprender las ideas y argumentos que se encuentran en el texto (p. I47-I48).

Las citadas reglas permiten al investigador tomar una postura científica frente al fenómeno, la cual se caracteriza por la aceptación de opiniones diferentes a la suya. Las reglas sirven también para que el historiador mire críticamente su propio trabajo y comprenda que la hermenéutica es un proceso que realmente nunca termina (Ricœur, 2000).

Handelsman (20I7) -quien elaboró su texto sobre metodología de la historia a principios del siglo XX-considera que el investigador tiene que empezar por la clasificación de las fuentes, proceso que divide en dos partes:

a) Fuentes indirectas: aquí pertenecen los documentos destinados a preservar la memoria del pasado en forma oral, por ejemplo leyendas, canciones, cuentos, anécdotas e imágenes (mapas, esculturas, planos, fotos, cuadros). También escritos como las antiguas inscripciones, memorias o diarios.

b) Fuentes directas: estas son los restos físicos de la existencia humana en el pasado. Entran en la clasificación las excavaciones, monumentos, tumbas e inscripciones. Así mismo los vestigios morales como memorias del pasado que viven en la 
colectividad, en las costumbres, creencias religiosas, prensa, arte, etc.

Handelsman se preocupó también por la crítica de las fuentes, que divide igualmente en dos partes:

a) La crítica externa (erudita) donde el rol es investigar en qué ambiente se creó la fuente y si es auténtica y original. El historiador tiene que dedicar tiempo para averiguar la fecha de creación de la fuente, el lugar, el autor y la autenticidad. El lugar de pertenencia es de las primeras actividades que permite seguir adelante. Para la averiguación de la fecha de creación de la fuente son importantes los criterios externos como apariencia física, tipo de escritura, material y otras características adicionales.

b) La crítica interna consiste en la investigación del contenido de la fuente y en la verificación de su utilidad para esclarecer el problema abordado. Aquí la parte más importante es la interpretación que se hace de su contenido y la obtención de toda la información que nos pueda transmitir. Una condición para hacer una buena interpretación es el conocimiento amplio del idioma, de la época o periodo, del ambiente y la sociedad y el objetivismo relativo.

En la crítica interna cabe la verificación de la veracidad de la fuente. Esta tiene que conducir al descubrimiento de información falsa o distorsionada. Involucra la calificación de objetividad, dado que se debe revisar si el autor participó directa o indirectamente en los acontecimientos que describe, el tiempo transcurrido desde que sucedió el hecho hasta que fue escrito. 
La información de las fuentes, así como los datos del autor (personalidad, inteligencia, curiosidad, educación, habilidad para narrar) condicionan el punto de vista del investigador, llevándolo a una interpretación subjetiva. El historiador debe saber que en la fuente investigada existen distorsiones consientes de la información -que aparecer por diferentes razones- y la función del investigador es ubicarlas. Estas distorsiones de la información en la fuente corresponden a los sentimientos y emociones personales, tales como odio, amistad o simpatía con la persona, acontecimiento o idea descrita.

El historiador estudia las sociedades como eran en el pasado y no reduce su interés a un campo específico, como lo hacen otras ciencias sociales (sociología, psicología, antropología). Su tarea es contribuir "al acercamiento integral al estudio de la sociedad" (Topolski, I998, p. 5I7).

Una de las tareas importantes de un historiador es contribuir en la búsqueda y descubrimiento de las regularidades en la vida social. La otra función importante de la historia es ayudar al ser humano para que se conozca a sí mismo.

Si la ciencia histórica quiere ejecutar mejor sus funciones y de manera más responsable, debe cambiar adecuadamente. Igual que la Revolución Industrial de los siglos XVIII y XIX desvió el rumbo de la investigación histórica, la actual revolución técnica exige más cambios esenciales en los estudios históricos. La historia se encuentra ahora en reconstrucción y expansión de largo alcance en sus métodos. Sus instrumentos se hacen cada vez más precisos y debe utilizar -cada vez más- métodos y resultados de otras disciplinas, si es que quiere desempeñar sus tareas integradoras (Topolski, 1998, p.5I8). 


\section{Intento de la conclusión}

Entre los desafíos que presenta el siglo XXI para los investigadores están la divulgación mucho más rápida y común de los resultados de sus trabajos, lo efímero de sus hipótesis, la saturación del "mercado", la cantidad de investigadores -en algunos casos de calidad muy dudosaentre muchos otros. El uso de metodología adecuada, para el caso concreto que se estudia, parece ser una herramienta que permite -por lo menos- hacer una investigación más hábil y profunda, defender su punto de vista de forma más disciplinada y también expresar los conceptos e ideas en lenguaje científico.

La aceptación de nuevas estrategias de investigación ocurre solo cuando los propios historiadores son capaces de asimilarlas dentro de su experiencia investigadora; en nivel pragmático, copiando y ajustando los procedimientos metodológicos.

En el mundo actual la historia se conforma de manera muy compleja y diversa, propone un discurso con propósitos paradigmáticos, tiene que cooperar y co-actuar con varias disciplinas científicas para enriquecer su contenido y poder interpretar el pasado de manera más profunda.

Parece también indispensable la autocrítica y autoconciencia del propio historiador. Dentro de ella, lo importante es visualizar que la práctica de la investigación y la manera en que se obtiene el conocimiento sobre el pasado, son productos de la cultura, lugar, tiempo, valores y tradiciones. A un nivel más amplio, depende del paradigma histórico.

Las categorías de veracidad, objetividad, neutralidad, credibilidad, etcétera, necesitan sustentarse en la filosofía, epistemología y metodología de la historia. Es también importante no caer en la creencia que la metodología de la historia es una ciencia auxiliar. Se 
debe recordar que esta no propicia la reflexión sobre la práctica de la investigación histórica a nivel teórico. En la mayoría de los casos proporciona solo los instrumentos para hacerlo.

Para finalizar, Deleuze en una de las pláticas con Foucault dijo que "la teoría es como una caja con herramientas (...) tiene que ser útil, tiene que funcionar." ${ }^{\text {I3 }}$ (p. I75). Ya a los investigadores se les tiene que dejar la responsabilidad de los instrumentos que utilicen, al fin que el tiempo verifica todo.

\section{Referencias}

Bolaños de Miguel, A. M. (20II) Crítica de la historia, política emancipatoria y moralidades postmodernas: la obra de Keith Jenkins. ISEGORÍA. Revista de Filosofía Moral y Política, núm. 44, enero-junio, 2I7-238.

Borleigh, T.W. (1983). ¿Tiene la historia algún sentido?: Una crítica a la filosofía de la historia de Popper. México: Fondo de Cultura Económica.

Deleuze G., y Foucault, M. (1985). “Intelectualisci a wladza. Rozmowy miedzy Deleuze i Foucault (Intelectuales y poder. Diálogos entre Deleuze y Foucault)." Revista Mes literario, núm. IO-II, p.I75.

Domanska, E. (20I0). "Jakiej metodologia potrzebuje wspolczesna humanistyka?." Teksty drugie, núm. I-2, pp. 45-55.

136 Finley, M. I. (I982). The world of Odysseus. Estados Unidos: nyrb.

Gottschalk, L. (1950). Understanding History: A Primer of Historical Method. New York: Alfred A. Knopf.

Hendelsman, M. (20I7). Historyka. Polonia: Neriton. 
Hobsbawm, E. J. (1998). Sobre la historia. España: Crítica.

Hutrado, M. (s/f ) Fuentes primarias y secundarias en la construcción del conocimiento histórico. Disponible en: https://www.dgespe.sep.gob.mx/public/comunidades/historia/r ecursos/Fuentes\%2oPrimaria s\%2oHurtado.pdf

Jenkins, H. (2006). ¿Por qué la historia? Ética y Postmodernidad. México: Fondo de Cultura Económica.

Klappenbach, H. (20I4). "Cerca de la Metodología de Investigación en la Historia de la Psicología." PSYKHE, vol. 23, núm. I, I-I2, doi:Io.7764/psykhe.23.1.584.

Krüger, H. H., (2005). Wprowadzenie $w$ teorie i metody badawcze nauk o wychowaniu. Polonia: GWP.

Kuhn, T. (1962). La estructura de las revoluciones científicas, México: Fondo de Cultura Económica.

Łobocki, M. (2005). Metody i techniki badań pedagogicznych, Polonia: Impuls.

Popper, K. (1969). "La lógica de las ciencias sociales." En T. Adorno, K. Popper, R. Dahrendorf, J. Habermas, H. Albert \& H. Pilot, en La disputa del positivismo en la sociología alemana, pp. IOI-II9. España: Grijalbo.

Ricœur, P. (2000). La memoria, la historia, el olvido. Argentina: Fondo de Cultura Económica.

Sticker, B. (1964). Die Stellung der Geschichte der Naturwissenschaften im Rahmen unserer heutigen Universitäten. 
Rybinska, A. (2015). "Zagadnienie metodologii e badaniach historycznyc i medycznych -problem interdyscyplinarnosci." Studia z zakresu historii spoleczno-gospodarczej, t. $\mathrm{XV}$. http://dx.doi.org/I0.I8778/2080-83I3.I5.II

Steiner, G. (2006). En el castillo de Barba Azul. Barcelona: Gedisa.

Topolski, J. (1998). Metodologia historii. Polonia: PWN.

Wysocka, E. (2004). Hermeneutyka, w: T. Pilch (red.), Encyklopedia pedagogiczna XXI wieku, t. II, Polonia: WA „Żak”.

\footnotetext{
${ }^{\text {I }}$ Historiador polaco (I882-I945) medievista y metodólogo de la historia, murió en un campo concentración.

${ }^{2}$ Traduccion de autor.

${ }^{3}$ Historiador estadounidense radicado en Ingalterra, expero en historia de la antigua Grecia.

${ }^{4}$ Izabela Tkocz terminó la carrera universitaria en la Facultad de Historia de la Universidad de Adam Mickiewicz en Poznan (Polonia), donde en los años 6os y 7os se formó la Escuela de Metodología de la Historia, siendo su principal exponente Jerzy Topolski. Sus trabajos son reconocidos mundialmente y se tradujeron a varios idiomas entre los que se cuenta el español.

${ }^{5}$ Ciencia que estudia el funcionamiento de los mecanismos y las conexiones nerviosas de los seres vivos.

${ }^{6}$ Todas las citas -de Topolski- que se incorporan en este documento son traducciones del autor.

${ }^{7}$ Las teorías tienen que ver con la verdad, en la medida en que hay fenómenos que tienen algún tipo de isomorfismo, con los modelos que caracterizan matemáticamente a dichas teorías.

${ }^{8}$ Aportación del autor.

${ }^{9}$ Todas las citas -de Łobocki- que se incorporan en este documento son traducciones del autor.
} 
${ }^{\text {Io }}$ Filosofo y fenomenólogo polaco, alumno de Husserl.

${ }^{\text {II }}$ En griego inventar.

${ }^{12}$ Todas las citas -de Krüger - que se incorporan en este documento son traducciones del autor.

${ }^{13}$ Foucault dijo que quería que sus libros fueran como cajas de herramientas que se usan por quien la necesita en una disciplina determinada. Decía también que sus libros no los escribe para los lectores, solo para los usuarios. Intelectualisci a wladza. Rozmowy miedzy Deleuze i Foucault (Intelectuales y poder. Diálogos entre Deleuze y Foucault). Revista Mes literario 1985, IO-II, p.I75. Traducción de autor.

Este artículo se publica bajo una licencia de Creative Commons Reconocimiento-NoComercial 4.0 Internacional, y puede ser usados gratuitamente para fines no comerciales, dando los créditos a los autores y a la revista.

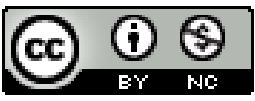


140 